\title{
Synthesis PID Controller \\ for Objects Second Order \\ with Regard to the Location Poles
}

\author{
Andrey P. Prokopiev*, \\ Vladimir I. Ivanchura and Rurik T. Emelyanov \\ Siberian Federal University \\ 79 Svobodny, Krasnoyarsk, 660041, Russia
}

Received 13.10.2015, received in revised form 26.12.2015, accepted 06.02.2016

The paper explored the method of synthesis of PID control systems for objects of the second order. As a theoretical basis of methods used modal method for linear systems with respect to the location of the complex poles. The obtained equations linking the values of the transient response, the complex poles and coefficients of the control object. The numerical illustration is given.

Keywords: automatic control system, synthesis, PID control, the modal method, the complex poles, transfer characteristic.

Citation: Prokopiev A.P., Ivanchura V.I., Emelyanov R.T. Synthesis pid controller for objects second order with regard to the location poles, J. Sib. Fed. Univ. Eng. technol., 2016, 9(1), 50-60. DOI: 10.17516/1999-494X-2016-9-1-50-60.

\section{Синтез ПИД-регулятора \\ для объектов второго порядка \\ с учетом расположения полюсов}

\author{
А.П. Прокопьев, \\ В.И. Иванчура, Р.Т. Емельянов \\ Сибирский федеральный университет \\ Россия, 660041, Красноярск, пр. Свободньий, 79
}

\begin{abstract}
Рассмотрена методика синтеза ПИД-регулятора системы управления для объектов второго порядка. В качестве теоретической основы методики использован модальный метод для линейных систем с учетом расположения комплексных полюсов. Получены соотношения, связывающие значения переходной характеристики, значения комплексных полюсов $и$ коэффициентов объекта управления. Приводится числовой пример.
\end{abstract}

(C) Siberian Federal University. All rights reserved

* Corresponding author E-mail address: prok1@yandex.ru 
Ключевые слова: система автоматического управления, синтез, ПИД-регулятор, модальный метод, комплексные полюсы, переходная характеристика.

\section{Введение}

Синтез регуляторов систем автоматического управления (САУ) - одна из основных предметных задач теории автоматического управления. Наибольшее распространение в САУ технологическими процессами получили пропорционально-интегрально-дифференцирующие (ПИД) регуляторы [1]. Одним из методов синтеза ПИД-регуляторов [2], активно развиваемым учеными, выступает модальный метод [3, 4 и др.]. В научных работах, посвященных синтезу регуляторов, отсутствуют соотношения, связывающие значения переходной характеристики и коэффициентов объекта управления с учетом расположения комплексных полюсов.

Наиболее распространенными для мобильных строительных и дорожных машин как объектов управления являются математические модели второго порядка.

\section{Исходное математическое описание системы и постановка задачи}

Рассматривается линейная система автоматического управления с передаточной функцией (ПФ) объекта второго порядка.

Передаточная функция объекта управления второго порядка $W_{o}(s)$ и ПИД-регулятора $W_{y}(s)$ имеет вид

$$
\begin{aligned}
& W_{o}(s)=\frac{b_{0} s+b_{1}}{a_{0} s^{2}+a_{1} s+a_{2}} ; \\
& W_{y}(s)=K_{p}+\frac{K_{i}}{s}+K_{d} s=\frac{K_{d} s^{2}+K_{p} s+K_{i}}{s},
\end{aligned}
$$

где $s$ - преобразователь Лапласа; $K_{p}, K_{i}, K_{d}$ - соответственно коэффициенты пропорциональности, интегрирования и дифференцирования.

При последовательном соединении ПФ объекта и ПИД-регулятора образуют разомкнутую систему с ПФ следующего вида:

$$
W(s)=W_{o}(s) W_{y}(s)=\frac{K_{d} b_{0} s^{3}+\left(K_{d} b_{1}+K_{p} b_{0}\right) s^{2}+\left(K_{i} b_{0}+K_{p} b_{1}\right) s+K_{i} b_{1}}{a_{0} s^{3}+a_{1} s^{2}+a_{2} s} .
$$

ПФ замкнутой системы управления с единичной обратной связью

$$
K(s)=\frac{W(s)}{1+W(s)}=\frac{K_{d} b_{0} s^{3}+\left(K_{d} b_{1}+K_{p} b_{0}\right) s^{2}+\left(K_{i} b_{0}+K_{p} b_{1}\right) s+K_{i} b_{1}}{\left(a_{0}+K_{d} b_{0}\right) s^{3}+\left(a_{1}+K_{d} b_{1}+K_{p} b_{0}\right) s^{2}+\left(a_{2}+K_{i} b_{0}+K_{p} b_{1}\right) s+K_{i} b_{1}} .
$$

Исходными данными для синтеза ПИД-регулятора САУ являются ПФ объекта управления $W_{o}(s)$ второго порядка и заданные показатели качества: перерегулирование и время регулирования.

Решается задача определения значений коэффициентов ПИД-регулятора $K_{d}, K_{p}, K_{i}$, обеспечивающих заданные показатели качества по заданным ПФ объекта управления второго порядка $W_{o}(s)$ и значениям действительного полюса $s_{1}=-\eta_{1}$ и комплексных полюсов $s_{2}=-\eta_{2}+j \beta$; $s_{3}=-\eta_{2}-j \beta$ ПФ замкнутой системы управления. 


\section{Вывод основных соотношений}

ПФ замкнутой системы с ПИД-регулятором при задании комплексных полюсов представим в виде

$$
K(s)=\frac{W(s)}{1+W(s)}=\frac{K_{d} b_{0} s^{3}+\left(K_{d} b_{1}+K_{p} b_{0}\right) s^{2}+\left(K_{i} b_{0}+K_{p} b_{1}\right) s+K_{i} b_{1}}{\left(a_{0}+K_{d} b_{0}\right)\left(s+\eta_{1}\right)\left(s+\eta_{2}+\beta j\right)\left(s+\eta_{2}-\beta j\right)} .
$$

Введем обозначения для упрощения выражения (1):

$$
b_{00}=\frac{K_{d} b_{0}}{a_{0}+K_{d} b_{0}} ; b_{01}=\frac{K_{d} b_{1}+K_{p} b_{0}}{a_{0}+K_{d} b_{0}} ; b_{02}=\frac{K_{i} b_{0}+K_{p} b_{1}}{a_{0}+K_{d} b_{0}} ; b_{03}=\frac{K_{i} b_{1}}{a_{0}+K_{d} b_{0}} .
$$

С учетом выражений (2) получим в области изображений для переходной характеристики замкнутой системы с ПИД-регулятором при задании значений комплексных полюсов:

$$
h(s)=\frac{b_{00} s^{3}+b_{01} s^{2}+b_{02} s+b_{03}}{\left(s+\eta_{1}\right)\left(s+\eta_{2}+\beta j\right)\left(s+\eta_{2}-\beta j\right) s} .
$$

Характеристическое уравнение замкнутой системы имеет третий порядок. При известных заданных действительном полюсе $s_{1}$ и комплексных полюсах $s_{2}, s_{3}$

$$
s_{1}=-\eta_{1} ; s_{2}=-\eta_{2}+\beta j ; s_{3}=-\eta_{2}-\beta j,
$$

это уравнение имеет вид

$$
\begin{aligned}
& D(s)=\left(s-s_{1}\right)\left(s-s_{2}\right)\left(s-s_{3}\right)=s^{3}+\left(\eta_{1}+2 \eta_{2}\right) s^{2}+\left[\eta_{1}\left(\eta_{2}-\beta j\right)+\right. \\
& \left.+\left(\eta_{2}+\beta j\right)\left(\eta_{1}+\eta_{2}-\beta j\right)\right] s+\eta_{1}\left(\eta_{2}-\beta j\right)\left(\eta_{2}+\beta j\right) .
\end{aligned}
$$

Применим упрощение

$$
\begin{aligned}
& \left.\eta_{1}\left(\eta_{2}-\beta j\right)+\left(\eta_{2}+\beta j\right)\left(\eta_{1}+\eta_{2}-\beta j\right)\right]=\beta^{2}+\eta_{2}^{2}+2 \eta_{1} \eta_{2}, \\
& \eta_{1}\left(\eta_{2}-\beta j\right)\left(\eta_{2}+\beta j\right)=\eta_{1}\left(\beta^{2}+\eta_{2}^{2}\right) .
\end{aligned}
$$

Тогда характеристическое уравнение примет следующий вид:

$$
D(s)=s^{3}+\left(\eta_{1}+2 \eta_{2}\right) s^{2}+\left(\beta^{2}+\eta_{2}^{2}+2 \eta_{1} \eta_{2}\right) s+\eta_{1}\left(\beta^{2}+\eta_{2}^{2}\right) .
$$

Характеристическое уравнение корректируемой системы можно представить таким выражением:

$$
\begin{aligned}
& \frac{\left(a_{0}+K_{d} b_{0}\right) s^{3}+\left(a_{1}+K_{d} b_{1}+K_{p} b_{0}\right) s^{2}+\left(a_{2}+K_{i} b_{0}+K_{p} b_{1}\right) s+K_{i} b_{1}}{a_{0}+K_{d} b_{0}}= \\
& =s^{3}+\left(\frac{a_{1}+K_{d} b_{1}+K_{p} b_{0}}{a_{0}+K_{d} b_{0}}\right) s^{2}+\left(\frac{a_{2}+K_{i} b_{0}+K_{p} b_{1}}{a_{0}+K_{d} b_{0}}\right) s+\frac{K_{i} b_{1}}{a_{0}+K_{d} b_{0}} .
\end{aligned}
$$

Приравнивая коэффициенты при одинаковых степенях $s$, получим:

$$
-52-
$$




$$
\begin{aligned}
& \frac{a_{1}+K_{d} b_{1}+K_{p} b_{0}}{a_{0}+K_{d} b_{0}}=\eta_{1}+2 \eta_{2} ; \\
& \frac{a_{2}+K_{i} b_{0}+K_{p} b_{1}}{a_{0}+K_{d} b_{0}}=\beta^{2}+\eta_{2}^{2}+2 \eta_{1} \eta_{2} ; \\
& \frac{K_{i} b_{1}}{a_{0}+K_{d} b_{0}}=\eta_{1}\left(\beta^{2}+\eta_{2}^{2}\right) .
\end{aligned}
$$

Определим установившееся значение $h_{s t a b}$ переходной характеристики $h(t)$ при задании комплексных полюсов в соответствии с теоремой предельного перехода:

$$
\lim _{s \rightarrow 0}(s \cdot h(s)) \rightarrow \frac{K_{i} b_{1}}{\eta_{1}\left(\eta_{2}-\beta j\right)\left(\eta_{2}+\beta j\right)\left(a_{0}+K_{d} b_{0}\right)}
$$

тогда установившееся значение $h_{s t a b}$

$$
h_{\text {stab. }}=\frac{K_{i} b_{1}}{\eta_{1}\left(\eta_{2}-\beta j\right)\left(\eta_{2}+\beta j\right)\left(a_{0}+K_{d} b_{0}\right)} .
$$

Упростив выражение (5), получим

$$
h_{\text {stab. }}=\frac{K_{i} b_{1}}{\eta_{1}\left(\eta_{2}^{2}+\beta^{2}\right)\left(a_{0}+K_{d} b_{0}\right)} .
$$

В соответствии с (4)

$$
\frac{K_{i} b_{1}}{a_{0}+K_{d} b_{0}}=\eta_{1}\left(\eta_{2}^{2}+\beta^{2}\right)
$$

подставляя (7) в (6), имеем

$$
h_{\text {stab. }}=\frac{K_{i} b_{1}}{\eta_{1}\left(\eta_{2}^{2}+\beta^{2}\right)\left(a_{0}+K_{d} b_{0}\right)}=\frac{\eta_{1}\left(\eta_{2}^{2}+\beta^{2}\right)}{\eta_{1}\left(\eta_{2}^{2}+\beta^{2}\right)}=1 .
$$

Полученный результат (8) подтверждает, что рассматриваемая замкнутая система управления является астатической относительно входного воздействия.

Определим начальное значение $h_{\text {stab. }}$ переходной характеристики $h(t)$ в соответствии с теоремой предельного перехода для случая задания комплексных полюсов:

T. e

$$
\lim _{s \rightarrow \infty}(s \cdot h(s)) \rightarrow \frac{K_{d} b_{0}}{a_{0}+K_{d} b_{0}},
$$

$$
h_{\text {init. }}=\frac{K_{d} b_{0}}{a_{0}+K_{d} b_{0}} .
$$

Доказано, что для рассматриваемой задачи синтеза ПИД-регулятора системы управления с ОУ второго порядка справедливо условие $h_{\text {init. }} \leq h(t) \leq 1$ при установившемся значении $h_{\text {stab. }}=1$. Это выражение не зависит от типа заданных полюсов. 
Таким образом, значение переходной характеристики замкнутой системы с ПИДрегулятором изменяется во времени от $h_{\text {init. }}$ до $h_{\text {stab. }}=1$. При этом если значение $b_{0}=0$, то начальное значение переходной характеристики $h_{\text {init. }}=0$.

Очевидно, что при некотором значении $b_{0}, h_{\text {init. }}$ войдет в $5 \%$-ю зону установившегося значения. Это свидетельствует о возможности значительного уменьшения времени переходного процесса при $b_{0}$ не равного 0.

\section{Определение значений составляющих переходной характеристики замкнутой системы управления}

Определим составляющие переходной характеристики $h(t)$ замкнутой системы с ПИДрегулятором в соответствии с теоремой предельного перехода, при задании комплексных полюсов в среде программы MathCAD применив функцию упрощения (simplify):

$$
\begin{aligned}
& \lim _{s \rightarrow-\eta_{1}}\left[\left(s+\eta_{1}\right) h(s)\right] \text { simplify } \rightarrow-\frac{\left(b_{1}-b_{0} \eta_{1}\right)\left(K_{d} \eta_{1}^{2}-K_{p} \eta_{1}+K_{i}\right)}{\eta_{1}\left(a_{0}+K_{d} b_{0}\right)\left(\beta^{2}+\eta_{1}^{2}-2 \eta_{1} \eta_{2}+\eta_{2}^{2}\right)} ; \\
& \lim _{s \rightarrow-\eta_{2}-\beta j}\left[\left(s+\eta_{2}+\beta j\right) h(s)\right] \text { simplify } \rightarrow \\
& -\frac{\left(b_{0} \eta_{2}-b_{1}+\beta b_{0} j\right)\left(K_{d} \eta_{2}^{2}-K_{p} \beta j-K_{d} \beta^{2}-K_{p} \eta_{2}+2 K_{d} \beta \eta_{2} j\right) j}{2 \beta\left(\eta_{2}+\beta j\right)\left(a_{0}+K_{d} b_{0}\right)\left(\eta_{2}-\eta_{1}+\beta j\right)} ; \\
& \lim _{s \rightarrow-\eta_{2}+\beta j}\left[\left(s+\eta_{2}-\beta j\right) h(s)\right] \text { simplify } \rightarrow \\
& \frac{\left(b_{1}-b_{0} \eta_{2}+\beta b_{0} j\right)\left(K_{d} \eta_{2}^{2}-2 j K_{d} \beta \eta_{2}-K_{d} \beta^{2}-K_{p} \eta_{2}+K_{i}+K_{p} \beta j\right) j}{2 \beta\left(\eta_{2}-\beta j\right)\left(a_{0}+K_{d} b_{0}\right)\left(\eta_{1}-\eta_{2}+\beta j\right)} .
\end{aligned}
$$

Обозначим:

установившееся значение $h_{\text {stab. }}$ в соответствии с (6)

$$
h_{\text {stab. }}=\frac{K_{i} b_{1}}{\eta_{1}\left(\eta_{2}^{2}+\beta^{2}\right)\left(a_{0}+K_{d} b_{0}\right)},
$$

при подстановке исходных данных получим установившееся значение $h_{\text {stab. }}$ переходной характеристики $h_{\text {stab. }}=1$, тогда составляющая $h_{s_{1}}(t)$ обусловленная полюсом $s_{1}=-\eta_{1}$,

$$
h_{s_{1}}(t)=-\frac{\left(b_{1}-b_{0} \eta_{1}\right)\left(K_{d} \eta_{1}^{2}-K_{p} \eta_{1}+K_{i}\right)}{\eta_{1}\left(a_{0}+K_{d} b_{0}\right)\left(\beta^{2}+\eta_{1}^{2}-2 \eta_{1} \eta_{2}+\eta_{2}^{2}\right)} \cdot e^{-\eta_{1} t},
$$

составляющая $h_{s_{2}}(t)$, обусловленная комплексным полюсом $s_{2}=-\eta_{2}+j \beta$,

$$
h_{s_{2}}(t)=-\frac{\left(b_{0} \eta_{2}-b_{1}+\beta b_{0} j\right)\left(K_{d} \eta_{2}^{2}-K_{p} \beta j-K_{d} \beta^{2}-K_{p} \eta_{2}+2 K_{d} \beta \eta_{2} j\right) j}{2 \beta\left(\eta_{2}+\beta j\right)\left(a_{0}+K_{d} b_{0}\right)\left(\eta_{2}-\eta_{1}+\beta j\right)} \cdot e^{\left(-\eta_{2}-\beta j\right) t},
$$

составляющая $h_{s_{3}}(t)$, обусловленная комплексным полюсом $s_{3}=-\eta_{2}+j \beta$,

$$
\begin{gathered}
h_{s_{3}}(t)=\frac{\left(b_{1}-b_{0} \eta_{2}+\beta b_{0} j\right)\left(K_{d} \eta_{2}^{2}-2 j K_{d} \beta \eta_{2}-K_{d} \beta^{2}-K_{p} \eta_{2}+K_{i}+K_{p} \beta j\right) j}{2 \beta\left(\eta_{2}-\beta j\right)\left(a_{0}+K_{d} b_{0}\right)\left(\eta_{1}-\eta_{2}+\beta j\right)} e^{\left(-\eta_{2}+\beta j\right) t} . \\
-54-
\end{gathered}
$$


Таким образом, для комплексных полюсов выражение переходной характеристики $h(t)$ примет следующий вид:

$$
h(t)=h_{s t a b .}+h_{s_{1}}(t)+h_{s_{2}}(t)+h_{s_{3}}(t),
$$

или, упростив выражение (12), заменив сумму $h_{s_{2}}(t)+h_{s_{3}}(t)$ на удвоенную действительную часть $2 \operatorname{Re}\left[h_{s_{2}}(t)\right]$ или $2 \operatorname{Re}\left[h_{s_{3}}(t)\right]$, т. е.

$$
h(t)=h_{s t a b .}+h_{s_{1}}(t)+2 \operatorname{Re}\left[h_{s_{2}}(t)\right], \text { или } h(t)=h_{s t a b .}+h_{s_{1}}(t)+2 \operatorname{Re}\left[h_{s_{3}}(t)\right] .
$$

Графическая реализация проверки примененного упрощения представлена на рис. 1: принятые обозначения $h_{12}(t)=2 \operatorname{Re}\left[h_{s_{2}}(t)\right]$ или $h_{13}(t)=2 \operatorname{Re}\left[h_{s_{3}}(t)\right]$.

\section{Синтез параметров пид-регулятора}

Равенства (4) представим в виде системы уравнений, в которой неизвестными являются значения коэффициентов ПИД-регулятора

$$
\begin{aligned}
& K_{d}\left[b_{1}-b_{0}\left(\eta_{1}+2 \eta_{2}\right)\right]+K_{p} b_{0}=a_{0}\left(\eta_{1}+2 \eta_{2}\right)-a_{1} ; \\
& -K_{d} b_{0}\left(\beta^{2}+\eta_{2}^{2}+2 \eta_{1} \eta_{2}\right)+K_{p} b_{1}+K_{i} b_{0}=a_{0}\left(\beta^{2}+\eta_{2}^{2}+2 \eta_{1} \eta_{2}\right)-a_{2} ; \\
& -K_{d} b_{0} \eta_{1}\left(\beta^{2}+\eta_{2}^{2}\right)+K_{i} b_{1}=a_{0} \eta_{1}\left(\beta^{2}+\eta_{2}^{2}\right) .
\end{aligned}
$$

Введем обозначения для представления системы уравнений в матричной форме:

$$
\begin{aligned}
& \lambda_{11}=b_{1}-b_{0}\left(\eta_{1}+2 \eta_{2}\right) ; \lambda_{12}=b_{0} ; \lambda_{13}=0 ; c_{1}=a_{0}\left(\eta_{1}+2 \eta_{2}\right)-a_{1} ; \\
& \lambda_{21}=-b_{0}\left(\beta^{2}+\eta_{2}^{2}+2 \eta_{1} \eta_{2}\right) ; \lambda_{22}=b_{1} ; \lambda_{23}=b_{0} ; c_{2}=a_{0}\left(\beta^{2}+\eta_{2}^{2}+2 \eta_{1} \eta_{2}\right)-a_{2} ; \\
& \lambda_{31}=-b_{0} \eta_{1}\left(\beta^{2}+\eta_{2}^{2}\right) ; \lambda_{32}=0 ; \lambda_{33}=b_{1} ; c_{3}=a_{0} \eta_{1}\left(\beta^{2}+\eta_{2}^{2}\right) .
\end{aligned}
$$

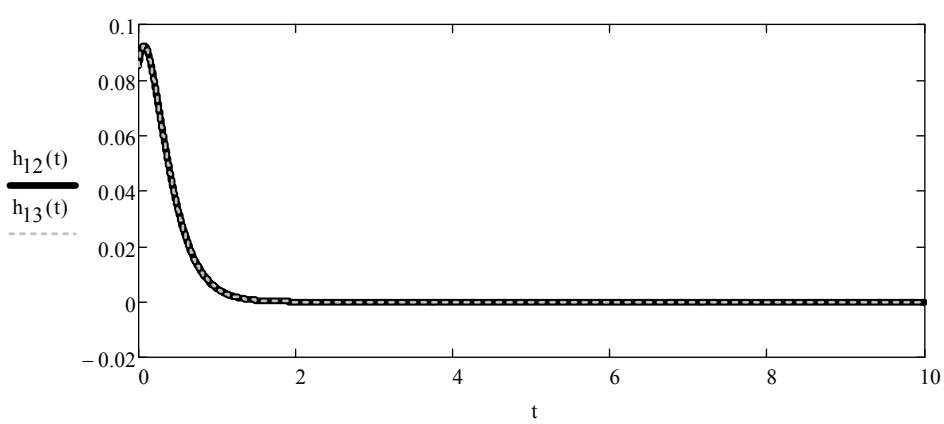

Рис. 1. Графическая реализация упрощения замены суммы $h_{s_{2}}(t)+h_{s_{3}}(t)$ на удвоенную действительную часть $2 \operatorname{Re}\left[h_{s_{2}}(t)\right]$ или $2 \operatorname{Re}\left[h_{s_{3}}(t)\right]$ 
С учетом принятых обозначений (14) сформируем матрицы:

$$
\Lambda=\left(\begin{array}{lll}
\lambda_{11} & \lambda_{12} & \lambda_{13} \\
\lambda_{21} & \lambda_{22} & \lambda_{23} \\
\lambda_{31} & \lambda_{32} & \lambda_{33}
\end{array}\right) ; C_{2}=\left(\begin{array}{c}
c_{1} \\
c_{2} \\
c_{3}
\end{array}\right) ; K_{2}=\left(\begin{array}{c}
K_{d} \\
K_{p} \\
K_{i}
\end{array}\right)
$$

Матричное уравнение для определения искомых значений коэффициентов регулятора имеет вид

$$
\left(\Lambda \cdot K_{2}\right)=C_{2}
$$

А его решение представляется в виде

$$
K_{2}=\Lambda^{-1} C_{2} \text {. }
$$

Система уравнений (13) значительно упрощается, если значение коэффициента $b_{0}=0$ :

$$
\begin{aligned}
& \frac{a_{1}+K_{d} b_{1}}{a_{0}}=\eta_{1}+2 \eta_{2} ; \\
& \frac{a_{2}+K_{p} b_{1}}{a_{0}}=\beta^{2}+\eta_{2}^{2}+2 \eta_{1} \eta_{2} ; \\
& \frac{K_{i} b_{1}}{a_{0}}=\eta_{1}\left(\beta^{2}+\eta_{2}^{2}\right) .
\end{aligned}
$$

В этом случае уравнения для расчета значений коэффициентов ПИД-регулятора имеют вид

$$
\begin{aligned}
& K_{d}=\frac{c_{1}}{b_{1}}=\frac{a_{0}\left(\eta_{1}+2 \eta_{2}\right)-a_{1}}{b_{1}} ; \\
& K_{p}=\frac{c_{2}}{b_{1}}=\frac{a_{0}\left(\beta^{2}+\eta_{2}^{2}+2 \eta_{1} \eta_{2}\right)-a_{2}}{b_{1}} ; \\
& K_{i}=\frac{c_{3}}{b_{1}}=\frac{a_{0} \eta_{1}\left(\beta^{2}+\eta_{2}^{2}\right) b_{1}}{b_{1}}
\end{aligned}
$$

Получены выражения для расчета коэффициентов ПИД-регулятора, включающие коэффициенты ПФ объекта второго порядка при заданных значениях комплексных полюсов. Выполнена проверка рассмотренной методики на конкретном примере.

Пример. Задан объект управления второго порядка с ПФ

$$
\begin{aligned}
W_{o}(s)=\frac{b_{0} s+b_{1}}{a_{0} s^{2}+a_{1} s+a_{2}}= & \frac{0,01 s+0,7}{0,04 s^{2}+0,12 s+1} . \\
& -56-
\end{aligned}
$$


Требуется синтезировать ПИД-регулятор, который обеспечит переходную характеристику без перерегулирования, а время регулирования $t_{p}=3 \mathrm{c}$.

Для реализации метода синтеза применены зависимости (15), (17). Расчеты выполнены в среде программы MathCAD.

Исходные данные для комплексных значений полюсов:

значение $\eta_{1}$ определено с учетом желаемого времени регулирования, равного 3 , с и расчета по известной приближенной формуле [5, 6]

$$
t_{\mathrm{p}} \approx \frac{3}{\operatorname{Re}(s)}, \eta_{1}=\operatorname{Re}(s)
$$

где $\operatorname{Re}(s)$ - действительная часть полюса, наиболее приближенного к мнимой оси корневой плоскости; значение $\eta_{2}$ и $\beta$ определяется из требуемого значения перерегулирования и влияния этого полюса на полюс $\eta_{1}$

$$
\eta_{1}=1 ; \eta_{2}=5 ; \beta=0,5 ; b_{1}=0,7 ; b_{0}=0,01 ; a_{0}=0,04 ; a_{1}=0,12 ; a_{2}=1,0 .
$$

Для заданных комплексных полюсов определены коэффициенты ПИД-регулятора:

$$
K_{2}=\left(\begin{array}{l}
K_{d} \\
K_{p} \\
K_{i}
\end{array}\right)=\left(\begin{array}{l}
0,528 \\
0,828 \\
1,633
\end{array}\right) ; K_{d}=0,528 ; K_{p}=0,828 ; K_{i}=1,633
$$

Выполнено моделирование переходного процесса для периода времени $t=0,0.001 \ldots 10$.

Составляющие $h_{s 1}(t), h_{s 2}(t), h_{s 3}(t)$, обусловленные полюсом $s_{1}=-\eta_{1}$ и комплексными полюсами $s_{2}=-\eta_{2}+\beta j ; s_{3}=-\eta_{2}+\beta j$, определяются по зависимостям (9) - (11).

Переходная характеристика $h(t)$ получена по выражению (12). При $t=1$ получим $h(t)=h_{\text {stab. }}+h_{s_{1}}(t)+h_{s_{2}}(t)+h_{s_{3}}(t)=0,117$.

Начальное значение $h_{\text {init. }}$ переходной характеристики $h(t)$

$$
h_{\text {init. }}=\frac{K_{d} b_{0}}{a_{0}+K_{d} b_{0}}=0,117
$$

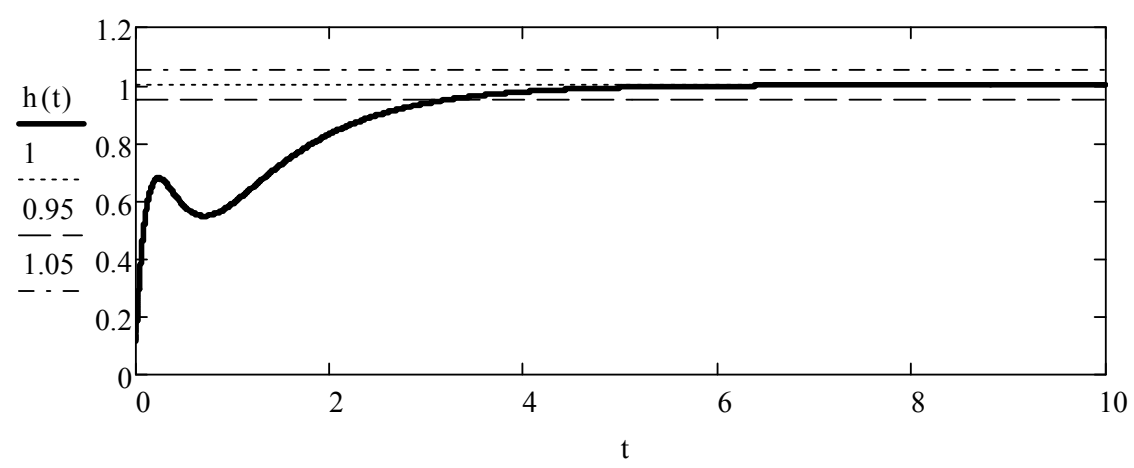

Рис. 2. График переходного процесса синтезированной системы с заданными комплексными полюсами $t_{p}=3$ с при $b_{0}=0,01$ 
На рис. 2 представлен график переходного процесса $h(t)$ замкнутой САУ с синтезированными параметрами ПИД-регулятора.

При известном значении $b_{0}=0,01$, заданных параметрах объекта второго порядка и комплексных полюсов в рассмотренном примере время переходного процесса, исходя из условия $5 \%$ зоны установившегося значения, равно 3 с.

Определены коэффициенты ПИД-регулятора при $b_{0}=0,05$ :

$$
K_{2}=\left(\begin{array}{l}
K_{d} \\
K_{p} \\
K_{i}
\end{array}\right)=\left(\begin{array}{l}
1,098 \\
3,106 \\
3,423
\end{array}\right) ; K_{d}=1,098 ; K_{p}=3,106 ; K_{i}=3,423 .
$$

Начальное значение $h_{\text {init. }}=0,579$ переходной характеристики $h(t)$ при $b_{0}=0,05$.

На рис. 3 представлен график переходного процесса $h(t)$ замкнутой САУ с синтезированными параметрами ПИД-регулятора при значении коэффициента объекта $b_{0}=0,05$.

При известном значении $b_{0}=0,05$, заданных параметрах объекта второго порядка и комплексных полюсов в рассмотренном примере время переходного процесса, исходя из условия $5 \%$ зоны установившегося значения, равно 2,45 с.

Определены коэффициенты ПИД-регулятора при $b_{0}=0,1$ :

$$
K_{2}=\left(\begin{array}{l}
K_{d} \\
K_{p} \\
K_{i}
\end{array}\right)=\left(\begin{array}{l}
5,420 \\
24,878 \\
20,992
\end{array}\right) ; K_{d}=5,420 ; K_{p}=24,878 ; K_{i}=20,992
$$

Начальное значение $h_{\text {init. }}=0,93$ переходной характеристики $h(t)$ при $b_{0}=0,1$

На рис. 4 представлен график переходного процесса $h(t)$ замкнутой САУ с синтезированными параметрами ПИД-регулятора при значении коэффициента объекта $b_{0}=0,1$.

В соответствии с разработанной методикой модального синтеза ПИД-регулятора для объектов второго порядка переходный процесс носит апериодический характер. При известном значении $b_{0}=0,1$, заданных параметрах объекта второго порядка и комплексных полюсов в рассмотренном примере время переходного процесса, исходя из условия 5 \% зоны установившегося значения, равно $0 \mathrm{c}$.

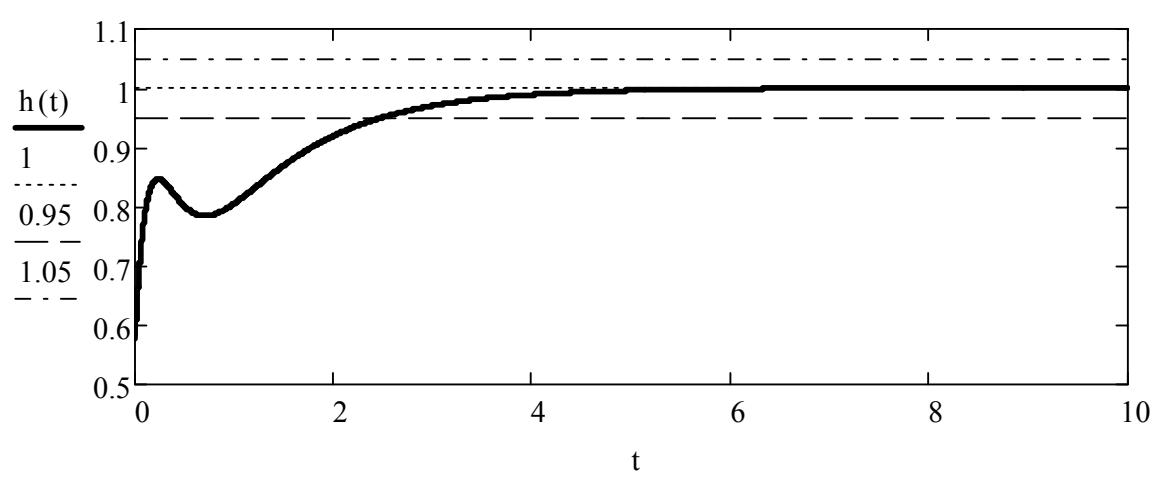

Рис. 3. График переходного процесса синтезированной системы с заданными комплексными полюсами $t_{p}=2,45$ с при $b_{0}=0,05$ 


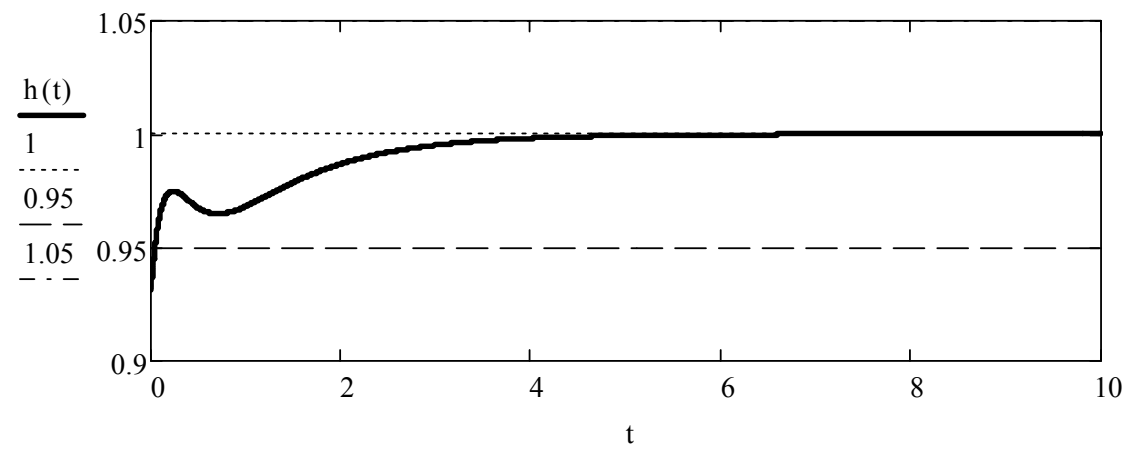

Рис. 4. График переходного процесса синтезированной системы с заданными комплексными полюсами $t_{p}=0$ с при $b_{0}=0,1$

Определение коэффициента $b_{0}$ передаточной функции объекта по заданному начальному значению $h_{\text {init. }}$ переходной характеристики $h(t)$

Преобразовав зависимость (18), получим следующее выражение для расчета $b_{0}\left(h_{\text {init. }}\right)$ :

$$
b_{0}\left(h_{\text {init. }}\right)=\frac{a_{0}}{\left(\frac{1}{h_{\text {init. }}}-1\right) K_{d}}, K_{d}=\frac{a_{0}\left(\eta_{1}+2 \eta_{2}\right)-a_{1}}{b_{1}},
$$

тогда итоговая зависимость примет вид

$$
b_{0}\left(h_{\text {init. }}\right)=-\frac{a_{0} b_{1}}{\left(\frac{1}{h_{\text {init. }}}-1\right)\left[a_{1}-a_{0}\left(\eta_{1}+2 \eta_{2}\right)\right]} .
$$

При заданном начальном значении переходной характеристики $h_{\text {init. }}=0,95$ величина коэффициента равна $b_{0}\left(h_{\text {init. }}\right)=1,6625$.

Зависимость (19) позволяет выполнить расчет коэффициента $b_{0}$ передаточной функции объекта по заданному начальному значению $h_{\text {init. }}$ переходной характеристики $h(t)$, коэффициентов ПФ объекта $\left(b_{1}, a_{0}, a_{1}\right)$, заданных полюсов $\left(\eta_{1}, \eta_{2}\right)$ системы управления. Рекомендации по определению значений комплексных полюсов могут быть предложены после дополнительных исследований по разработанной методике.

\section{Заключение}

Разработана методика модального синтеза ПИД-регулятора по заданным значениям параметров объекта второго порядка и комплексных полюсов замкнутой системы управления.

Исследование показало, что при некотором значении $b_{0}$, не равного 0 , начальное значение переходной характеристики $h_{\text {init. }}$ войдет в $5 \%$-ю зону установившегося значения. Это свидетельствует о возможности значительного увеличения быстродействия скорректированной системы. 


\section{Список литературы}

[1] O’Dwyer A. PI and PID controller tuning rules: an overview and personal perspective, Proceedings of the IET Irish Signals and Systems Conference. Dublin Institute of Technology, June, 2006, 161-166; http://arrow.dit.ie/engscheleart/39.

[2] Прокопьев А.П., Иванчура В.И., Емельянов Р.Т. Идентификация нелинейной системы управления с ПИД-регулятором. Труды Х Международной конференции «Идентификаиия систем и задачи управления» (SICPRO‘15), М.: Институт проблем управления им. В.А. Трапезникова PAН, 2015, 387-397; http://www.sicpro.org/sicpro15/code/r15_08.htm [Prokop'ev A.P., Ivanchura V.I., Emel'ianov R.T., Identification of nonlinear control systems with the PID control, SICPRO'15, Moscow, 2015, 387-397; http://www.sicpro.org/sicpro15/ code/r15_08.htm (in Russian)]

[3] Вадутов О.С. Синтез ПИД-регулятора в системах с запаздыванием методом условной оптимизации с ограничениями на размещение полюсов. Известия Том. политех. ун-та. Информационные технологии, 2014, 325(5), 16-22. [Vadutov O.S. Synthesis of PID controller in timedelay systems by method of constrained optimization with restrictions on placement of poles, Bulletin of the Tomsk Polytechnic University. Information technology, 2014, 325(5), 16-22 (in Russian)]

[4] Ефимов С.В., Замятин С.В., Гайворонский С.А. Синтез ПИД-регулятора с учетом расположения нулей и полюсов системы автоматического регулирования. Известия Том. политех. yн-ma, 2010, 317(5), 102-107 [Efimov S.V., Zamiatin S.V., Gaivoronckii S.A. Synthesis of the PID controller with respect to the location of zeros and poles of the system of automatic control, Bulletin of the Tomsk Polytechnic University, 2010, 317(5), 102-107 (in Russian)]

[5] Ефимов С.В., Гайворонский С.А., Замятин С.В. Задачи корневого анализа и синтеза и синтеза систем автоматического управления. Известия Том. политех. ун-та, 2010, 316(5), 1620 [Efimov S.V., Gaivoronckii S.A., Zamiatin S.V. Root tasks of analysis and synthesis and synthesis of automatic control systems, Bulletin of the Tomsk Polytechnic University, 2010, 316(5), 16-20 (in Russian)]

[6] Удерман Э.Г. Метод корневого годографа в теории автоматических систем. М.-Л.: Госэнергоиздат, 1963, 112 c. [Underman E.G. Root locus method in the theory of automatic systems, Moscow-leningrad, Gosenergoizdat, 1963, 112 p. (in Russian)] 